\title{
Detector Concepts for the Electron-Ion Collider
}

\author{
José Repond ${ }^{1}$ \\ Argonne National Laboratory \\ 9700 S Cass Avenue, Lemont, IL 60439, U.S.A. \\ E-mail: repondeanl.gov
}

This paper reviews the major detector concepts being developed for the future Electron-Ion Collider (EIC). After a brief introduction into the EIC, some of the choices and options for a detector for the EIC are reviewed, followed by a discussion of the four major concepts to date: BEAST, JLEIC, ePHENIX, and TOPSiDE. Particular emphasis is given to novel technologies, such as imaging calorimetry, precision timing silicon sensors, and streaming readout.

13th International Workshop in High pT Physics in the RHIC and LHC Era (High-pT2019)

19-22 March 2019

Knoxville, Tennessee, USA

\section{${ }^{1}$ Speaker}




\section{Introduction: the Electron-Ion Collider}

The Electron-Ion Collider (EIC) is a planned nuclear physics facility. The facility will collide unpolarized or polarized electrons/positrons with unpolarized or polarized protons or ions. It is worthwhile to recall that the EIC will not be the first such facility, as this honor has already been bestowed on HERA which initiated operation in 1992. Table I compares some of the major parameters of the two machines. Most notable is the relatively low center-of-mass energy, the higher luminosity (by two orders of magnitude), and the capability to circulate ions of the EIC. The scientific goal of the EIC is the detailed study of both the perturbative and the non-perturbative aspects of Quantum Chromodynamics (QCD). One of the most important goals is the precision tomography of the nucleon and of nuclei, the study of contributions to the nucleon spin, and the search for and possible discovery of gluon saturation effects.

Two national laboratories have been identified as possible sites for the EIC: Brookhaven National Laboratory proposes to add an electron ring to the existing RHIC machine (currently colliding ions); Jefferson Lab proposes to use CEBAF as electron injector and to add an electron storage ring and an ion injector complex and storage ring.

\begin{tabular}{|l|l|l|}
\hline & HERA & EIC \\
\hline Operation & $1992-2007$ & $>2030$ \\
\hline Electrons/positrons & $\begin{array}{l}27.5 \mathrm{GeV} \\
\text { (Polarized) }\end{array}$ & $\begin{array}{l}5-18 \mathrm{GeV} \\
\text { Polarized }\end{array}$ \\
\hline Protons & $820-920 \mathrm{GeV}$ & $60-250 \mathrm{GeV}$ \\
& Not polarized & Polarized \\
\hline Ions & Not available & $\begin{array}{l}\text { Up to } 100 \mathrm{GeV} / \mathrm{u} \\
\text { Polarized }\end{array}$ \\
\hline$\sqrt{s_{e p}}$ & & $35-134 \mathrm{GeV}$ \\
\hline Luminosity & $300-318 \mathrm{GeV}$ & $>10^{34} \mathrm{~cm}^{-2} \mathrm{~s}^{-1}$ \\
\hline Colliding beam detectors & $10^{32} \mathrm{~cm}^{-2} \mathrm{~s}^{-1}$ & Subject of this talk \\
\hline Fixed target experiments & HERMES, HERAB & $?$ \\
\hline
\end{tabular}

Table I. Comparison of some of the parameters of HERA and the EIC. To date no fixed target experiments have been proposed for the EIC.

The EIC enjoys the unanimous support of the nuclear physics community. The 2015 Long Term Plan for Nuclear Science [1] identified the EIC as the highest priority for a future facility. Recently, the National Academy of Sciences completed its review [2] of the proposed facility with a glowing endorsement. The Department of Energy is expected to grant CD0 (mission need) within the next few months, thus turning the EIC into a (funded) project.

\section{Measurements at the EIC}

To understand the challenges facing the design of EIC detectors, it is useful to review the major physics topics to be addressed by the facility. The EIC features a broad 
physics program requiring the precision measurment of the scattered electron, the identification of pions and kaons, and the measurement of hadronic jets, among others. Table II summarizes the most important physical quantities to be measured, the processes used to do so, and the specific challenges from the point of view of detection and measurement. Note the importance of pion-kaon separation which is seen to be essential for a number of different topics.

\begin{tabular}{|l|l|l|}
\hline Physical quantity & Process & Measurement challenges \\
\hline Structure Functions $\mathrm{F}_{2}{ }^{\prime} \mathrm{F}_{\mathrm{L}}$ & Inclusive scattering & $\begin{array}{l}\text { Electron identification, } \\
\text { hadron measurement, } \\
\text { background rejections, } \\
\text { luminosity }\end{array}$ \\
\hline Spin structure functions & Inclusive scattering & $\begin{array}{l}\text { Same as above and } \\
\text { polarization, pion/kaon } \\
\text { separation }\end{array}$ \\
\hline Gluon density & Charm, dijet production & $\begin{array}{l}\text { Secondary verteces, } \\
\text { pion/kaon separation, } \\
\text { hadronic jets }\end{array}$ \\
\hline $\begin{array}{l}\text { Generalized Parton } \\
\text { Distributions (GPDs) }\end{array}$ & $\begin{array}{l}\text { Deeply Virtual Compton } \\
\text { Scattering (DVCS) }\end{array}$ & $\begin{array}{l}\text { Forward proton, electron, } \\
\text { photon measurements, } \\
\text { background rejection }\end{array}$ \\
\hline $\begin{array}{l}\text { Transverse Momentum } \\
\text { Distributions (TMDs) }\end{array}$ & $\begin{array}{l}\text { Semi-inclusive Deep } \\
\text { Inelastic Scattering }\end{array}$ & $\begin{array}{l}\text { Same as for structure } \\
\text { functions and pion/kaon } \\
\text { separation }\end{array}$ \\
\hline $\begin{array}{l}\text { Nuclear Parton Distribution } \\
\text { Functions (PDFs) }\end{array}$ & Scattering on nuclei & $\begin{array}{l}\text { Same as for structure } \\
\text { functions and detection of } \\
\text { nuclear fragments }\end{array}$ \\
\hline Photon PDFs & $\begin{array}{l}\text { Hadronic jet energy } \\
\text { resolution }\end{array}$ \\
\hline
\end{tabular}

Table II. Summary of the most important physics quantities to be measured at the EIC and their detection challenges.

\section{Reconstruction of the kinematic variables of deep inelastic scattering events}

Several methods exist to reconstruct the kinematics of deep inelastic scattering events, foremost being the so-called electron method (using the energy and angle of the scattered electron), double angle method (using the angles of the scattered electron and of the hadronic system), and the Jacquet-Blondel method (using the energy and angle of the hadronic system). The latter is the only applicable method for charged current interactions, as in these events the outgoing neutrino is not detected.

To illustrate the importance of the precise measurement of the hadronic system, consider the kinematic reconstruction based on the electron method. Here, the resolution in Bjorken $\mathrm{x}$ is given by

$$
\frac{\delta x}{x}=\frac{1}{y} \frac{\delta E_{e}}{E_{e}},
$$


where $y$ is the inelasticity parameter and $\mathrm{E}_{\mathrm{e}}\left(\delta \mathrm{E}_{\mathrm{e}}\right)$ is the energy (energy resolution) of the scattered electron. As can be seen, the resolution deteriorates rapidly with decreasing $\mathrm{y}$. On the other hand, a measurement of the hadronic system can provide a good estimate of Bjorken $\mathrm{x}$ at low $\mathrm{y}$

$$
\frac{\delta x}{x}=\frac{1}{1-y} \frac{\delta E_{q}}{E_{q}}
$$

where $E_{q}$ is the energy of the scattered quark. The precision, however, is proportional to the hadronic energy resolution. For more details on kinematic reconstruction at $e p$ colliders, see $[3,4]$.

\section{Design choices}

The development of concept detectors for the EIC faces several general design choices. Foremost is the choice between the design of a general purpose or a specialized detector. The former enables the study of a broad spectrum of physics topics and will most likely excel in background identification and rejection. However, this comes at the price of a more complex and difficult design. The remainder of the paper will cover the concepts for general purpose detectors only.

The beam energies at ep colliders are naturally very asymmetric. This led to asymmetric designs of $\mathrm{H} 1$ and ZEUS, the two multi-purpose detectors at HERA. In particular, for both the depths of the calorimeters in the electron direction were significantly reduced. However, with increased luminosity, particles of all species are emitted into all parts of the solid angle, necessitating full calorimetric coverage, also for hadrons. Furthermore, an asymmetric design generates strong mechancial forces on the solenoid, which ought to be avoided.

Finally, the cost of specific detector technologies varies with time. For instance, the cost per area of silicon decreased substantially over the past decades. Therefore, in the early stages of concept development, it is prudent not to overemphasize cost and not to eliminate currently costier options from the get go, as they might become economically viable a few years down the road.

\section{The Four Detector Concepts}

Currently, there are four distinct concepts for a general purpose EIC detector: BEAST, JLEIC, ePHENIX, and TOPSiDE. ePHENIX is conceived as evolving from the sPHENIX detector operating at RHIC [5]. The other three are not based on existing devices and will require assembly from scratch. In the following, we will cover these four concepts in more detail. 


\section{BEAST}

The BEAST (Brookhaven eA Solenoidal Tracker) concept is being developed by Brookhaven National Laboratory. A cut-out view of the detector is shown in Fig. 1. The overall length of the detector is approximately nine meters.

Tracking of charged particles will be performed by a vertex detector and a TimeProjection-Chamber (TPC), supplemented by micromegas both in the barrel and the endcap regions. The vertex detector consists of four layers in the barrel region and three disks in the forward and backward regions. The vertex detector features Monolithic Active Pixel Sensors (MAPS) with $20 \times 20 \mu \mathrm{m}^{2}$ pixels and digital readout (i.e. 1-bit resolution per pixel). The TPC is about two meters long and is read out with Gas Electron Multipliers (GEMs). The thickness of the TPC endplates corresponds to $15 \%$ of a radiation length, whereas the field cage corresponds to $4-5 \%$ of a radiation length.

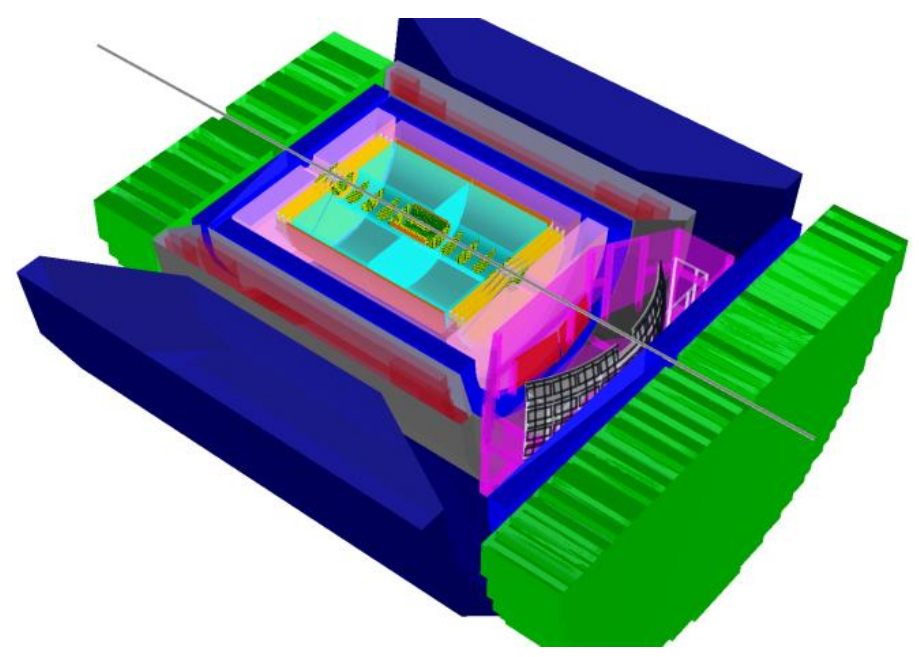

Figure 1. Cut-out view of the BEAST detector concept: TPC (cyan), electromagnetic calorimeter (blue), hadronic calorimeter (green), Ring Imaging Čerenkov counters (pink), superconducting solenoid (grey and red), return yoke (dark blue).

The calorimeter is segmented in depth into an electromagnetic and a hadronic section. The preferred solution for the electromagnetic section utilizes scintillating fibers embedded in a Tungsten powder matrix. Tests of calorimeter prototypes in the Fermilab test beam showed an excellent energy resolution of $(7-10) \% / \sqrt{E}$ for electrons between 1 and $14 \mathrm{GeV}$. In the forward and rear direction the electromagnetic calorimeter is supplemented by a hadron calorimeter based on scintillator plates and lead absorbers. The very backward direction $(\eta<-2)$ is covered by a matrix of high resolution $(\sim 2 \% / \sqrt{E})$ $\mathrm{PbWO}_{4}$ crystals.

BEAST lacks a barrel hadron calorimeter. The decision to omit hadron calorimetry in the barrel region might have to revisited, as there are several reasons why complete calorimetric coverage (hermeticity) is important: kinematic reconstruction needs the measurement of all hadrons (double angle method); the kinematics of charged current events can only be reconstructed using the hadronic system (Jacquet-Blondel method); 
electron identification is needed in the barrel region and is greatly helped by a hadron calorimeter; jet physics requires the measurement of all hadrons, etc. In general, precision physics will require hermeticity of the calorimetric coverage.

Particle identification (i.e. pion-kaon-proton separation) is perfomed using different techniques depending on the momentum of particles. At low momenta $(p<1$ $\mathrm{GeV} / \mathrm{c}$ ) $\mathrm{dE} / \mathrm{dx}$ or time-of-flight (with modest time resolution) can be applied. In the barrel and in the forward region, a Ring Imaging Čerenkov (RICH) with Aerogel (diffractive index $\sim 1.5$ ) and gas (diffractive index $\sim 1$ ), respectively, will be deployed. Both devices will be located in front of the calorimeter.

\section{JLEIC}

The major features of the JLEIC concept being developed at Jefferson Lab are similar to the ones of the BEAST concept. One notable difference is that JLEIC omits the hadron calorimeter in the backward (electron) direction, but features hadron calorimetry in the barrel region, see Fig. 2.

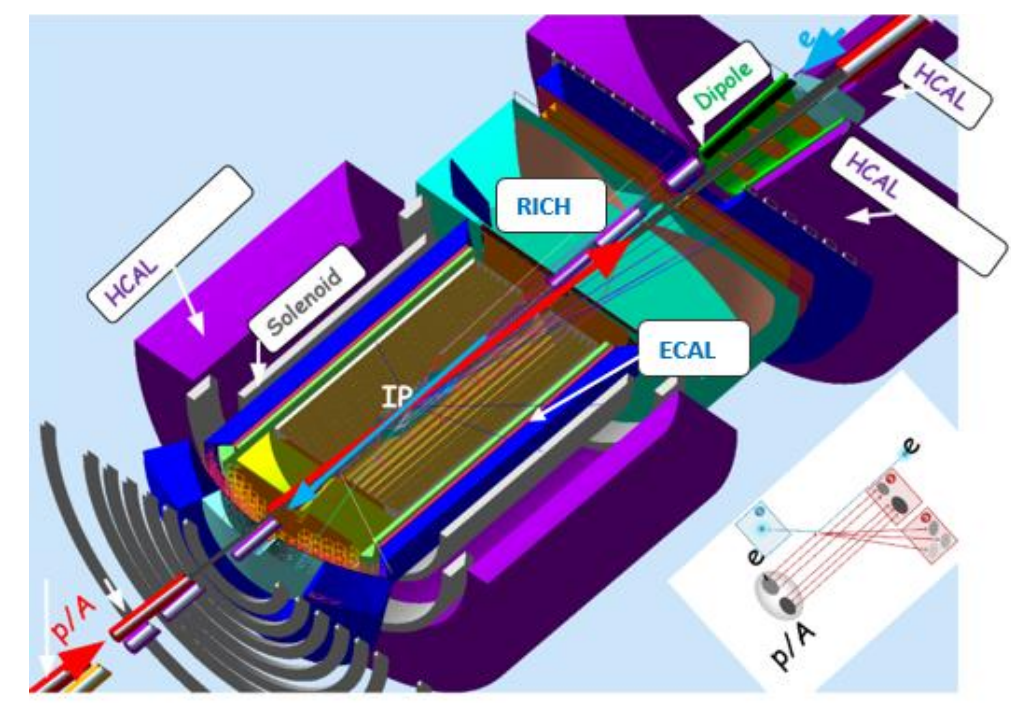

Figure 2. Cut-out view of the JLEIC detector concept.

This concept group initiated detailed studies of the instrumentation of the very forward and backward regions. Several devices are necesssary in the backward region: a $0^{0}$ photon calorimeter for the luminosity measurement via the Bethe-Heitler process, a Low- $\mathrm{Q}^{2}$ tagger measuring low-energy electrons deflected by the first dipole magnet, and, for the electron beam polarization measurement, a Compton photon calorimeter and an electron tracking detector combined with a beam chicane involving a set of four dipole magnets. The other concepts are likely to adopt similar solutions to the very forward/backward detector regions. 


\section{8. $\quad$ ePHENIX}

Again, the ePHENIX concept is similar to BEAST, featuring the same type of tracking and calorimetry. A cost saving will be the reuse of the Babar magnet with a 1.5 Tesla field. For more details on sPHENIX, the precursor to ePHENIX, see [5].

\section{TOPSiDE}

The TOPSiDE (Time Optimized Silicon Detector for the EIC) takes advantage of the many developments in detector paradigms and technology of the last two decades, in particular of the progress having been made in the context of the International Linear Collider (ILC). The aim is to realize a detector whose output is a list of identfied particles and their momenta, similar to what is obtained at the hadron level in Monte Carlo simulations of physics processes, such as deep inelastic scattering. In other words, the detector will identify and measure each particle (charged or neutral) individually, which in turn requires imaging calorimetry with very fine segmentation both laterally and longitudinally [6]. In addition, particle identification will be performed by time-of-flight measurements in the calorimeter itself, requiring sensors with timing resolutions of the order of ten picoseconds. In this concept, the calorimeter measures not only the energy, but also the precise location $(\mathrm{x}, \mathrm{y}, \mathrm{z})$ and time of the energy deposits (five dimensions or 5D). Figure 3 shows a cut-out view of TOPSiDE. In the forward direction the detector includes a gaseous RICH, a dipole magnet together with precision tracking using silicon strip detectors, similar in design to the other concepts.

TOPSiDE features a vertex detector and a pure silicon tracker, surrounded by a hermetic 5D calorimeter. The electromagnetic calorimeter is of the sandwich type with silicon sensors interleaved by tungsten plates. The silicon sensors are of the Low-GainAvalanche-Diode (LGAD) type with ultra-fast timing [7]. The imaging hadron calorimeter utilizes either small scintillator pads $\left(3 \times 3 \mathrm{~cm}^{2}\right)$ or Resistive Plate Chambers with $1 \times 1 \mathrm{~cm}^{2}$ readout pads interleaved with steel absorber plates. The superconducting coil is placed on the outside of the calorimeter, thus avoiding interference with calorimetric measurements. This elegant solution minimizes the amount of material in front of the calorimeter and renders devices such as preshower detectors, transition radiation detectors, or Čerenkov counters in front of the calorimeter redundant. Due to the imaging capabilities of the calorimeter, a muon identification system is also made redundant.

Advantages of imaging calorimetry are many. Charged particle identification (electron - muon - hadron separation) becomes almost trivial, due to their distinct interactions resulting in electromagnetic or hadronic showers or single tracks. The fine granularity allows for the identification of electromagnetic subshowers in hadronic showers and consequently for the application of software compensation techniques [8]. Measurements of energy deposits in the last layers of the calorimeter can be utilized to correct for longitudinal leakage, thus improving the single particle energy resolution. Through the identification of track segments in the calorimeter, the gain of individual channels or of areas of the calorimeter can be monitored in situ [9]. At hadron colliders, contributions from the underlying events can be identified and subtracted. However, the 
most important advantage concerns the application of so-called Particle Flow Algorithms (PFAs) $[6,10]$. The latter attempt to measure the energy/momentum of particles using the detector subsystem providing the best resolution. Thus, charged particle momenta are measured with the tracker, whereas photons (neutral hadrons, i.e. neutrons and $K_{L}^{0}$ ) are measured with the electromagnetic (entire) calorimeter. This approach leads to a significant improvement in jet energy resolution and is being adopted by upgrades of the LHC experiments. The EIC environment is particularly suited for the application of PFAs, due to the low particle multiplicity and low center-of-mass energy.

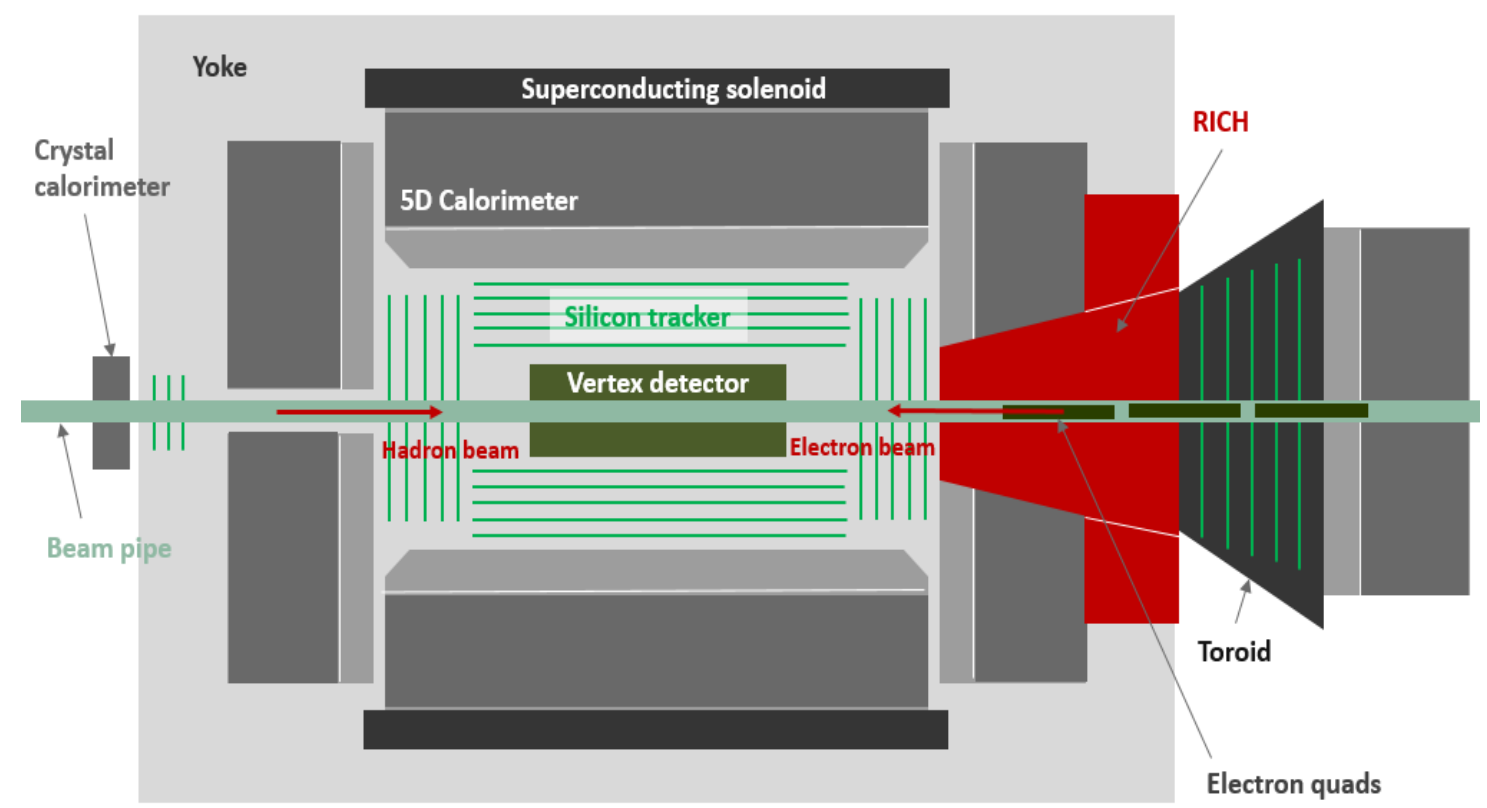

Figure 3. Side view of the TOPSiDE detector concept.

\section{Ultra-fast silicon and the PENTACAL}

As noted above, particle identification (pion-kaon-proton separation) will be achieved through time-of-flight measurements in the calorimeter. At the EIC, for most of the solid angle, particle momenta do not exceed seven $\mathrm{GeV} / \mathrm{c}$. Detailed simulation showed that particle identification in this moment range requires timing resolutions of the order of ten picoseconds [11].

A vigorous, worldwide effort is ongoing to develop ultra-fast silicon detectors [7]. Currently the best performance, $\sigma_{t} \sim 18$ picosecond, has been achieved with $35 \mu \mathrm{m}$ thick LGAD sensors [12]. Efforts are under way to implement the front-end readout including the digitization onto each individual pixel with the prospect of further improving the timing resolution. The near-term goal is to assemble a prototype electromagnetic calorimeter with 5D technology, featuring one wafer per layer and approximately twenty layers. For obvious reasons, this prototype calorimeter has been dubbed the PENTACAL. 


\section{Streaming readout}

The detector concepts are investigating the option of a streaming readout. With a streaming readout the complete information from all detector subsystems is available for selecting events to be written to disk/tape. The selection is performed online by a set of tunable algorithms. The major advantage of this approach is the elimination of the traditional hardware trigger path and in the process greatly simplifying the data acquisition system. Studies are under way to understand the implications of this approach for the choice of detector subsystems and their readout/data schemes. For more information on the streaming readout, see [13].

\section{Conclusions}

With the strong support of the nuclear physics community, the present author is confident that the Electron-Ion Collider will happen. A starting date for physics operation of 2030 is conceivable.

The physics environment of the EIC poses a number of challenges for the detector design. These challenges are being addressed by four distinct detector concepts: BEAST, JLEIC, ePHENIX, and TOPSiDE. Within this context, several novel detector technologies are being pursued, such as supplementing the central solenoid with a forward dipole for improved momentum measurement, a forward Ring Imaging Čerenkov Detector, 5D calorimetry (imaging calorimetry with ultra-fast silicon sensors), and streaming readout, among others.

\section{Acknowledgements}

The author thanks the organizers for inviting him to this most stimulating workshop.

\section{References}

[1] https://science.energy.gov/ /media/np/nsac/pdf/2015LRP/2015_LRPNS_091815.pdf

[2] https://www.nap.edu/catalog/25171/an-assessment-of-us-based-electron-ion-colliderscience

[3] J. Feltesse, Measurement of inclusive differential cross sections, Proceedings of the HERA workshop, Hamburg, Germany (1987).

[4] S. Bentvelsen, J. Engelen, P. Koojiman, Reconstruction of $\left(x, Q^{2}\right)$ and extraction of structure functions in neutral current scattering at HERA, Proceedings of the 'Physics at HERA' workshop, Hamburg, Germany (1991).

[5] R. Reed, these proceedings.

[6] F. Sefkow et al., Experimental tests of particle flow calorimetry, Rev. of Modern Physics 88, 15003 (2016). 
[7] H. F. W. Sadrozinski et al., 4D tracking with ultra-fast silicon detectors, Rept. Prog. Phys. 81 (2018), no.2, 026101.

[8] C. Adloff et al., Hadronic energy resolution of a highly granular scintillator-steel hadron calorimeter using software compensation techniques, JINST 7 (2012) P09017.

[9] C. Adloff et al., Track segments in hadronic showers in a highly granular scintillatorsteel hadron calorimeter, JINST 8 (2013) P09001.

[10] M. A. Thomson, Particle flow calorimetry and the PandoraPFA algorithm, Nucl. Instr. Meth. A611 (2009) 25-40.

[11] J. Repond, TOPSiDE: Concept of an EIC detector, PoS DIS2018 (2018) 179.

[12] H. F. W. Sadrozinski, Measurement of timing precision of ultra-fast silicon detectors, talk given at the Workshop on 'Picosecond timing detectors for physics and medical applications', Torino, Italy (2018).

[13] https://eic.jlab.org/wiki/index.php/Streaming_Readout_II 